\title{
Routing and Re-Routing in a LEO/MEO Two-tier Mobile Satellite Communications System with ][nter-Satellite Links
}

\author{
Jian-Hao Hu Kwan L. Yeung \\ Department of Electronic Engineering \\ City University of Hong Kong \\ Hong Kong, PRC \\ E-mail: \{jhhu, kyeung\}@ee.cityu.edu.hk \\ Tao Li \\ Institution of Information and System \\ University of Electronic: Science and Technology of China \\ Cheng Du, si Chuan; 610054, PRC
}

\begin{abstract}
A novel LEO/MEO two-tier satellite communication system with inter-satellite links (ISLs) is proposed for providing multimedia services to global mobile users. This two-tier system architecture can reduce the transmission delay for long-distance users via MEO satellites while keeping the benefits of using LEO satellites as the service access nodes. The routing and re-routing during a handoff operation is simplified. Since the physical topoiogy of the underlying network is time-dependent, routing is crucial for guaranteeing the delay and delay variation performance for interactive applications. In this paper, we decompose the routing problem into two parts, routing in the access network and routing in the core MEO ISL network. For access network, a new routing algorithm called Maximum Holding Access Protocol (MHAP) is proposed for minimizing the number of LEO handoffs. For core MEO ISL network, both Minimum Transmission Delay Routing (MTDR) and Minimum Transmission Time Jitter Routing (MTTJR) are investigated. Using computer simulations, we show that the proposed routing algorithms can reduce the probability of call rerouting and thus are very suitable for providing interactive multimedia services.
\end{abstract}

\section{Introduction}

The next generation mobile satellite communications systems will provide flexible and high-quality multimedia services to users at anywhere and at anytime. These satellites will have powerful onboard processing and switching capabilities. Sub-communication networks with inter-satellite links (ISLs) in the space segment can also be established. The traditional Geostationary Earth Orbit (GEO) satellites are not suitable for this purpose because of the long transmission delay and high propagation loss. Instead, the Low Earth Orbit (LEO) and Medium Earth Orbit (MEO) satellites will be adopted in the next generation satellite systems. Teledesic, Sativod, Celestri, Sky-bridge and M-star systems [1] are some proposed examples.

Compared with the GEO satellites, the altitude of the LEO satellites is much lower. Thus the propagation loss and transmission delay are greatly reduced $[8,9]$. This makes the hardware implementations of both mobile terminals and satellites relatively easier. On the other hand, a LEO satellite system needs a larger number of satellites to provide global coverage. Establishing sub-networks in the space segment using ISLs (inter-satellite links) is a very complicated issue. Owing to the time varying physical topology of the ISL networks, routing in the space segment network is very difficult to manage [7]. Compared with the LEO system, the MEO system needs fewer satellites in the constellation to provide global coverage, and the ISL network is much simpler. As a result, it is easier to conduct routing and handoff in MEO systems. If a sender is far away from the receiver (e.g., one user is at Beijing, while the other is at New
York), the total transmission delay using MEO systems will be less than using LEO systems.

In [2,3], schemes based on the LEO/GEO two-tier satellite architecture have been proposed as a candidate for next generation satellite communications systems to provide multimedia services. But the problem of long transmission delay caused by GEO satellites have not been solved. In this paper, we propose a novel LEO/MEO two-tier architecture for supporting multimedia communications. It consists of $16 \mathrm{MEO}$ satellites in the upper tier and 63 LEO satellites in the lower tier. As we are going to show in the rest of the paper, the proposed architecture has the following major advantages:

(a) simplicity in managing the space networks formed by timevarying ISLs,

(b) reduction in call re-routing probability for end-to-end connections, and

(c) reduction in transmission delay for users using MEO satellites.

For the proposed two-tier architecture, routing is crucial for guaranteeing the quality of service $(\mathrm{QoS})$ requirement on delay and delay variation for interactive applications because the underlying network topology is time-dependent. Therefore we focus on designing efficient routing algorithms in this paper.

In the next section, we present the two-tier LEO/MEO satellite system in details. In Section III, we divide the routing problem into two parts, at the access network and at the core MEO ISL network. A routing protocol called Maximum Holding Access Protocol (MHAP) is designed for minimizing the number of handoffs in the access network. For the core MEO ISL network, two routing schemes based on Dijkstra algorithm are investigated. One is the Minimum Transmission Delay Routing (MTDR) and the other is Minimum Transmission Time Jitter Routing (MTTJR). In Section IV, simulations are conducted for evaluating the proposed routing schemes. We found that the proposed routing schemes can significantly reduce the call re-routing probability. This in turn cuts down the associated signaling traffic. Finally we conclude the paper in Section V.

\section{Two-tier Satellite Network Architecture}

The proposed two-tier LEO/MEO satellite system consists of 63 LEO satellites in the lower tier and 16 MEO satellites in the upper tier as shown in Fig. 1. The LEO satellites provide the global coverage to the mobile users and the MEO satellites provide the switching/routing capability for inter-satellite communications (LEO-to-MEO and MEO-to-MEO). To facilitate the establishment of the inter-satellite links (ISLs), Walker constellation [4] is used in this architecture. Using Walker constellation, the topological relationship among satellites in the same tier can be maintained. Thus the ISLs between any two MEO satellites can be set up easily. 


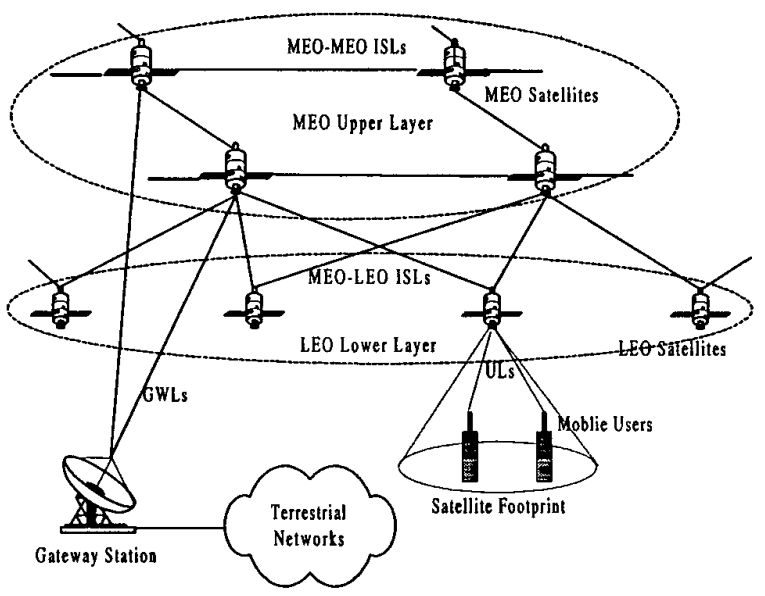

Fig.1 The architecture of the proposed two-tier satellite system

In our design, the Walker factors of the MEO and LEO constellations are $\mathrm{S} / \mathrm{P} / \mathrm{T} /=16 / 4 / 3$ and $\mathrm{S} / \mathrm{P} / \mathrm{T}=63 / 7 / 6$ respectively. Other constellation parameters used are listed in Table 1.

Table 1 Constellation parameters used by the proposed LEO/MEO system.

\begin{tabular}{|c|c|c|}
\hline & MEO & LEO \\
\hline Altitude(Km) & 10353 & 1400 \\
\hline Orbital Periodic(Minute) & 360 & 114 \\
\hline Number of Satellites & 16 & 63 \\
\hline Number of Planes & 4 & 7 \\
\hline Orbital Inclination (Degree) & 45 & 48 \\
\hline Number of ISLs in Same Plane & 2 & 0 \\
\hline Number of ISLs between Adjacent Plans & 2 & 0 \\
\hline Number of ISLs between Layers & $<=12$ & 2 \\
\hline
\end{tabular}

Consider Fig. 1. Each MEO satellite in the upper tier has four ISLs connected to four adjacent MEO satellites, two in the same orbital plane, and two in the adjacent orbital planes. This establishes a vertex-symmetric network. Each LEO satellite in the lower tier has two ISLs connected to two MEO satellites falling into its sight. There is no direct ISL links between any two LEO satellites. Using this architecture, a MEO satellite acts as a switching node in the network. It provides all necessary switching among MEO satellites, LEO satellites and the ground gateway stations. Mobile users are connected directly to a LEO satellite. It acts as the service access node with local switching capability. If two mobile users covering by two different LEO satellites, or one in the satellite system and the other in the terrestrial network, want to establish a connection, some MEO satellites must be involved in the end-to-end connection for providing the necessary call switching and routing.

In the proposed two-tier satellite architecture, there are four types of wireless links.

- Links between mobile users and LEO satellites, denoted by $U L s$. When a call is set up, an idle UL is assigned to a mobile user for its dedicated use until the call is over, or a handoff occurs. A handoff occurs when a mobile leaves the coverage of a LEO satellite where the mobile's current UL is connected. It involves the establishment of a new UL to a newly selected LEO satellite, and the release of the old UL from the old LEO satellite. From the routing point of view, a call re-routing is performed for each handoff. The way of selecting new satellite is covered in Section III.A.

- Inter-satellite links (ISLs) between LEO satellites and MEO satellites. These links are simultaneously shared by many mobile users/connections. They are removed and re-constructed on a regular basis as the satellites move along their respective orbits. At any moment, a LEO satellite is connected to two MEO satellites via two such ISLs. As the LEO satellite moves, one of the existing ISLs will be removed and a new ISL to another MEO will be established. The decision of which and when the next MEO satellite will be connected is predetermined from the Walker constellation. During such a handoff operation, all connections in the associated ISL will be switched/handed-off simultaneously.

- Inter-satellite links (ISLs) between any two MEO satellites. These links are fixed links because the Walker constellation is used. Each MEO satellite is always connected to the same four other adjacent MEO satellites using these links. This forms a fixed mesh network topology for all MEO satellites in the core MEO ISL network. Thus multiple paths/routes between any two MEO satellites exist and therefore routing becomes necessary.

- Links between $M E O$ satellites and ground gateway stations, denoted by GWLs. These links are shared by those connections with one communication end on the terrestrial network. A ground gateway station is used to relay the traffic between the terrestrial network and the space segment network.

With this two-tier constellation, many advantages can be obtained. First, the switch management in the ISL networks is simplified. As we are going to show in Section IV, the call re-routing probability is also reduced. Second, the LEO satellite management is simplified as there is no direct ISL between any two LEO satellites. All network routing functions involve MEO satellites. Third, for long-distance users, routing through MEO satellites can reduce the end-to-end transmission delay. Finally, the proposed LEO/MEO two-tier system has less stringent requirements on the automatic track \& point (ATP) antenna on LEO satellites for deploying ISLs. This is because the relative moving speed between a LEO and a MEO satellite is much lower than that between two L.EO satellites.

\section{Routing \& Re-Routing}

Since both LEO and MEO satellites are constantly moving in their own orbits, the distance between two satellites as well as the underlying physical network topology are time-dependent. Like a conventional cellular system, when a handoff occurs, a re-routing of an on-going call is necessary for maintaining a continuous connection. For the proposed two-tier system, two types of handoff exist.

- LEO handoff. A LEO handoff happens when the footprint of a LEO satellite can no longer cover a mobile user with an ongoing call. Then the mobile sets up a new UL to a LEO satellite whose footprint covers it and the old UL is removed. This is a handoff from one LEO to another LEO satellite, so we call it $L E O$ handoff. When a LEO handoff occurs, the newly selected/ connected LEO satellite needs to decide which of its two ISLs should be used to carry the on-going call. According to the MHAP routing strategy in the next sub-section, the ISL connected to the MEO satellite that provides a longer service time $^{1}$ to the associated LEO satellite will be selected.

\footnotetext{
${ }^{1}$ To distinguish the coverage time of a LEO satellite on a mobile user, we use the term service time for a LEO that is under the coverage of a MEO satellite where an ISL is connected.
} 
- MEO handoff. A LEO satellite always connects to two MEO satellites using two ISLs. As the LEO satellite moves out of the service area of a connected MEO satellite, it sets up a new ISL to another selected MEO and the old ISL is removed. We call this a $M E O$ handoff. A MEO handoff is different from a LIEO handoff in that (a) MEO handoff carries out on a regular and periodic basis according to the Walker constellation used; and (b) an ISL is shared by many on-going calls and thus a MIEO handoff involves the switching of all calls on the old ISL.

For providing continuous connection, both LEO and MEO handoffs should be transparent to end users. Not only to minimize the transmission delay and delay variation, a good call routing/re-routing scheme should also minimize the number of handoffs, which in turn can minimize the associated signaling traffic for handoffs.

Fig. 2 shows a typical call routing between two mobile users $A$ and B. User A is connected to satellites $\mathrm{S} 11, \mathrm{~S} 12$ and $\mathrm{S} 13$ at time t0, t1 and 2 , respectively. Similarly, user B is connected to $\$ 33$, S34 and $\mathrm{S} 35$ at time $\mathrm{t0}^{\prime}, \mathrm{t1} \mathbf{\prime}^{\prime}$ and $\mathrm{t2}$ '. At any given time shown in the figure, each LEO is connected to two MEO satellites via its two ISL links, ISLO \& ISL1. The end-to-end connection between users A and B consists of three segments: the access network from user $A$ to the MEO core network, the MEO core network, and the access network from MEO core network to user B.

If the receiving mobile is located in the same LEO footprint as the call originating mobile, then the call is routed/reflected back direstly to the earth without using ISL resources. Routing in such cases; is trivial. Studies in [11] show that for a typical LEO footprint diameter of $2000-3000 \mathrm{~km}$, about $85 \%$ traffic is within the same footprint. The remaining amount of (long distance) traffic involves some MEO satellites as shown in Fig. 2. In this paper, our focus is on how to efficiently handle routing for distant calls.

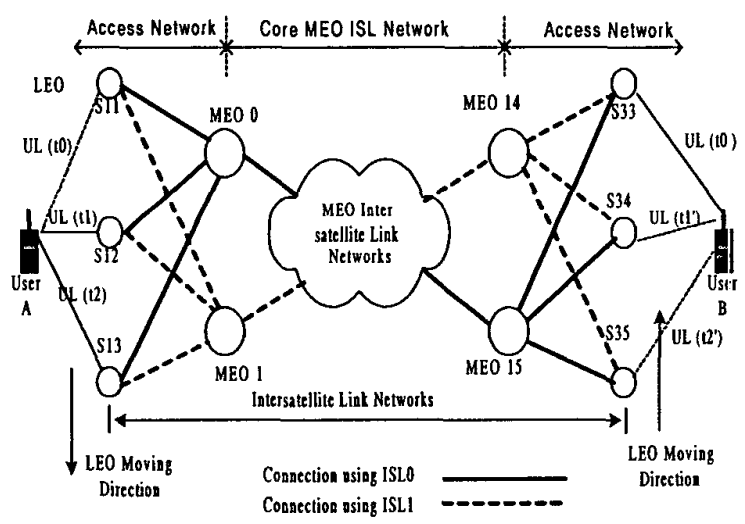

Fig. 2 Routing updates for two mobile users.

\section{A. Providing Routing \& Re-Routing at Access Network}

If a mobile user with an on-going call can always connect to the LEO satellite that provides the maximum coverage time for it, the call can be completed with the minimum number of LEO handoffs. It is clear that the LEO satellite that is the closest to the mobile user does not provide the maximum coverage time for it. In the following, a new routing protocol for minimizing the LEO handoffs at the access network, called Maximum Holding Access Protocol (MHAP), is proposed.

In the proposed two-tier architecture, it can be found that with a minimum allowable elevation angle of 10 degrees, the probability of at least two satellites simultaneously falling into a mobile user's sight is more than $90 \%$. According to the satellite calendar, the mobile can therefore always find the satellite that provides the maximum coverage time to it. If the destination user is located in another LEO footprint or in the terrestrial system, the LEO satellite will select one of the two connecting MEO satellites that provides a longer service time to it to support this call. The service time of a MEO satellite, which ranges from 30 to 50 minutes, is much longer than the LEO satellite coverage time for a mobile, which is only about 10 minutes. It can be found that when a MEO handoff occurs, the traffic load on the affected ISL link is zero. This is because before a MEO handoff happens, all active calls on the affected ISL have already been handed off to other LEO satellites during earlier LEO handoffs. This in turn is due to the facts that (a) the LEO handoff occurs at a much higher rate than MEO handoff, and (b) during each LEO handoff, the newly selected LEO always choose the MEO satellite that provides a longer service time to it to carry the call.

Owing to the Walker constellation adopted, when a LEO handoff occurs, the new LEO satellite will select the MEO satellite that is used by the pervious LEO satellite with a very high probability (more than $85 \%$ from our simulation results). Consequently, the reconstruction of the ISLs caused by LEO handoff mainly occurs between LEO and MEO satellites. The routing in the MEO core network will remain unchanged with a very high probability during call re-routing. This is very important for reducing the data transmission delay variation because the ISLs between MEO satellites account for the major portion of the end-to-end delay.

The procedures of Maximum Holding Access Protocol (MHAP) routing algorithm can then be summarized below:

(1) When a call is initiated, the mobile selects a LEO satellite that can provide the maximum coverage time to it among all LEO satellites in sight using the satellite calendar.

(2) If some MEO satellites are involved for a long distance call, the LEO satellite selected in Step (1) chooses (from the two connected MEO satellites) the MEO satellite that provides a longer service time to carry the call.

(3) When a LEO handoff occurs, repeat Steps (1) and (2).

\section{B. Providing Routing and Re-Routing at Core MEO ISL Network}

The Maximum Holding Access Protocol solves the routing problem in the access network. In this section, we focus on the routing in the core MEO ISL network. For a two-tier satellite system, the transmission delay is dominated by the propagation delay between MEO satellites in the core network. An efficient core network routing can minimize the end-to-end delay as well as the delay variations. Two routing schemes based on Dijkstra algorithm are investigated. One is the Minimum Transmission Delay Routing (MTDR); the other is Minimum Transmission Time Jitter Routing (MTTJR). MTDR is simply the minimum distance routing, whereas MTTJR focuses on minimizing the transmission delay variation. In general, the end-to-end transmission delay obtained using MTTJR is bigger than that of MTDR, but MTTJR is particularly suitable for interactive multimedia services because of its small delay variation.

A routing protocol is activated for finding a path in the core network whenever a new call is established, or an on-going call is re-routed (in case of a LEO handoff). When a new call arrives, the MHAP protocol is responsible for routing in the two access network segments. In the core MEO ISL network, a minimum-distance path is found for carrying the call. When a LEO handoff occurs, if the newly selected LEO satellite connects to the same MEO satellite as the previous LEO, the path in the core network is kept. In this case, only the access network segment is re-routed by MHAP. If the newly selected LEO satellite does not connect to the same MEO satellite as 
the previous LEO, the path in the core network needs to be rerouted. Using MTTJR, the path that has the minimum delay variation as compared to the original path during call set up will be selected. If MTDR is used, again the path with the minimum distance (at that moment) will be selected.

The movement of MEO and LEO satellites increases the routing complexity in the two-tier satellite architecture. However, this constellation movement is regular and periodic. We can divide the constellation period, which is the lowest common multiple of the MEO and LEO satellites rotational periods, into finite independent time slots of varying slot lengths. The network topology does not change during each slot. Then a routing table can be set up for each satellite during each slot. The routing tables can be pre-calculated by the ground station and downloaded to (or stored at) each satellite. This avoids the on-board satellite processing. According to the constellation movement, each satellite updates its routing table in each time slot.

In the studies of $[5,6]$, fixed length slot design is adopted. In order to guarantee the routing performance, the slot length is relatively small ( 10 seconds). Compared with the fixed slot design, the variable slot length design we proposed in this paper has the following advantages:

- Significant reduction in the number of routing tables because the number of slots is significantly reduced.

- The routing table is updated simultaneously with the topology re-construction of the ISL network.

- The peak traffic and ISL interference from background noises can be easily handled.

Although the routing table at each satellite is updated on a slot basis, as far as a particular end-to-end user connection is concerned, the rerouting is only performed when a LEO handoff occurs. To summarize, the procedures for end-to-end routing from mobile users $A$ to $B$ in a two-tier satellite system shown in Fig. 2 are:

(1) For each end user, select one LEO satellite and one MEO satellite at the access network for carrying the call using MHAP protocol.

(2) Find an optimal path through the MEO ISL core network using either MTDR or MTTJR.

(3) If user A or user B requests a LEO handoff, select a new LEO satellite for it using MHAP. If the newly selected LEO satellite uses the same MEO satellite as the previous LEO, the path in the MEO ISL core network remains unchanged; otherwise, find a new path in the core network using either MTDR or MTTJR.

\section{Simulation Results}

In this section, the performance of the routing protocols proposed in Section III is studied using computer simulations. In particular, a connection between two distant users is simulated, where one user is at Beijing, China and the other one is at New York, USA. Multiple hops in the core MEO ISL network is needed for this connection. In the simulation, the constellation operation is a continuous procedure, and the user access is random. A hybrid simulation method is used. The simulation length is two constellation periods. The multi-beam antenna and "cell-fixed" method are used in the simulation. Based on the study in [10], we know that the beam handoff is equal to satellite handoff in a cell-fixed system. Therefore, we focus only on the LEO satellite handoff.

The probability distribution function of the average transmission delay for the two users is shown in Fig. 3. The average transmission delay for using MTDR ranges from 120 to $320 \mathrm{~ms}$. The average transmission delay for MTTJR ranges form 200 to $360 \mathrm{~ms}$. The

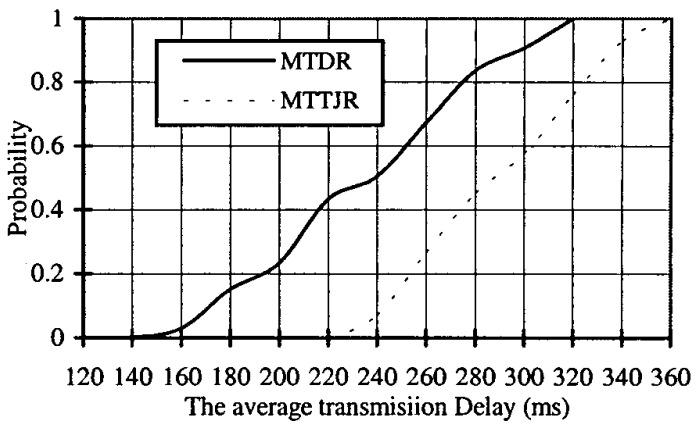

Fig. 3 The probability distribution function of the average transmission delay of MDTR and MTTJR

probabilities that average transmission delays are less than 300 and $320 \mathrm{~ms}$ are 0.91 and 0.999 respectively using MTDR. Using MTTJR, the corresponding probabilities are 0.93 and 0.999 . We can see from Fig. 3 that the average transmission delay of MTDR is smaller than MTTJR.

Next we consider the delay variation performance in Fig. 4 . The transmission delay variation is caused by two factors: (a) the timevarying distance between satellites, and (b) traffic following different paths caused by re-routing during a call. We call the former path variation $(P V)$ and the latter re-routing variation $(R V)$. The probability density functions (PDF) for these two variations are shown in Fig. 4 for both MTDR and MTTJR. The call holding time is exponentially distributed with mean 3 minutes. From Fig. 4, we can find that using MTDR, the probabilities of path variation less than 100 and $10 \mathrm{~ms}$ are 0.99 and 0.97 respectively, and the probabilities of re-routing variation less than 100 and $10 \mathrm{~ms}$ are 0.96 and 0.95 respectively. Using MTTJR protocol, the probabilities of the path variation less than 100 and $10 \mathrm{~ms}$ are 0.999 and 0.98 , and the probabilities of the re-routing variation less than 100 and $10 \mathrm{~ms}$ are 0.99 and 0.97 . We can see that the re-routing variation and thus the total transmission delay variation of MTTJR is less than that of MTDR. For supporting interactive multimedia services, a small transmission delay variation is essential.

Fig. 5 shows the performance of the total transmission delay variation (path variation plus re-routing variation) experienced by a random user with fixed call holding time of 2 minutes, 4 minutes and

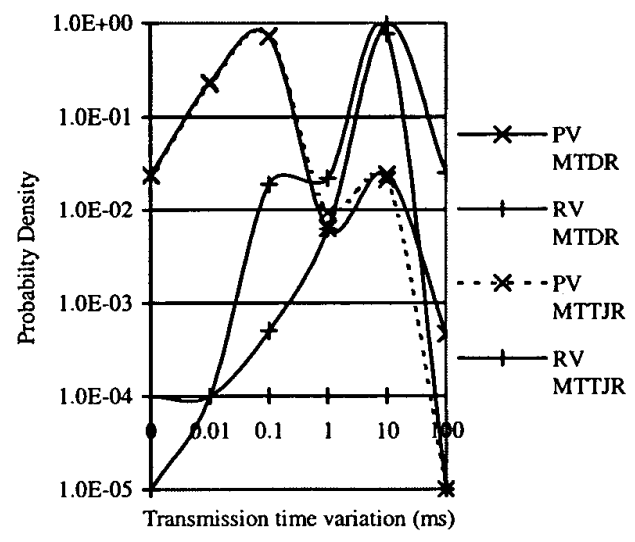

Fig. 4 The probability density function of path variation and rerouting variation using MTDR and MTTJR 
30 minutes, respectively. In our simulations, we assumed that a fixed size window slides through an orbital period. The size of the window is equal to the fixed call holding time. The transmission delay variations are then found from the collected transmission delays in each window. From Fig. 5 we can see that if the MTDR is used, the probabilities that the transmission variation is less than 90 ms are $0.999,0.975$ and 0.581 for window sizes (or call holding times) of 2, 4 and 30 minutes. For MTTJR, the probabilities that the transmission variation is less than $40 \mathrm{~ms}$ for the three call holding times are $0.99,0.98$ and 0.68 respectively. From these results, vve can see that (a) the longer the call duration, the higher the delay variation, and (b) using MTTJR, a mobile user experiences less transmission variation.

Fig. 6 shows the call re-routing probabilities. The call re-routing probability is defined as the probability that the call experiences at least one re-routing during its call holding time. As expected, we can see that the re-routing probability increases with the mean cill holding time (exponentially distributed). Besides, the re-routing probability using MTDR is a little bit less than that of MTTJR. A small re-routing probability implies less signaling traffic for rerouting calls. Compared with that in [7], the re-routing probabilities of using MTDR and MTTJR are smaller. This is because all reroutings in our proposed two-tier satellite system are caused by the LEO handoffs only.

\section{Conclusion}

Combining the advantages of LEO and MEO satellites, a novel LEO/MEO two-tier mobile satellite communications architecture with inter-satellite links was proposed in this paper. The routing and re-routing problem in the proposed two-tier satellite system has been studied. For routing in the access network, a new protocol called maximum holding access protocol was proposed. For routing in the MEO core network, two algorithms MDTR and MTTJR have been investigated. The simulation results showed that the proposed routing/re-routing schemes can reduce the probability of call rerouting and is therefore suitable for providing multimedia services for global mobile users.

\section{References:}

[1] Chen Ruming, "MEO/LEO Satellite Communication:s," Communications Science, Vol. 13, No.7, 44-48, 1997.

[2] Le Pond Chin et. al. , "Performance of Two Layer LEO Satellite Communication Network," IEEE Trans on Aerospace and Electronic Systems, Vol.33, No. 1, 225-231, 1997.

[3] Kazuhir Kimura and etal., "Double-Layered Inclined Orbit Constellation For Advanced Satellite Communications Network," IECE Tran. Commun. Vol. E80-B, No. 1, 93-102, 1997.

[4] Chia Jiu Wang, "Structural Properties of a Low Earth Orbit Satellite Constellation the Walker Delta Network," Globecom' 93, 93-102, 1993.

[5] Markus W, Cecilia D, Hans-Jorg V, "ATM- Based Routing in LEO/MEO Satellite Networks with Intersatellite Links," IEEE Journal on Selected Areas in Communications, Vol. 15, No.1, 69-82, January 1997.

[6] Markus Werner, Georg Berndl and Bernhard Edmaier, "Performance of Optimized Routing in LEO Intersatellite Link Networks," VTC'97. MC2-6, 1997.

[7] Gragopoulos, E. Papapetrou and F. N. Pavlidou, "Routing Techniques for Low Orbit Satellite Configurations," International Conference on Telecommunications' 98 , W28, 1998.

[8] S. Dimolitesas and J.G. Phipps, "Experimental Quantification of Voice Transmission Quality of Mobile Satellite Personal

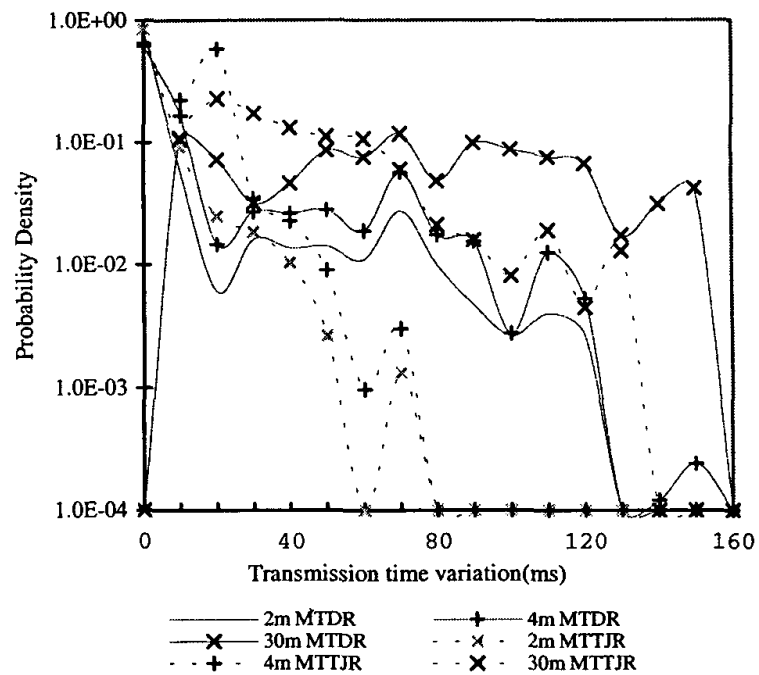

Fig. 5 The probability density function of the total end-to-end transmission time variation using MTDR and MTTJR.

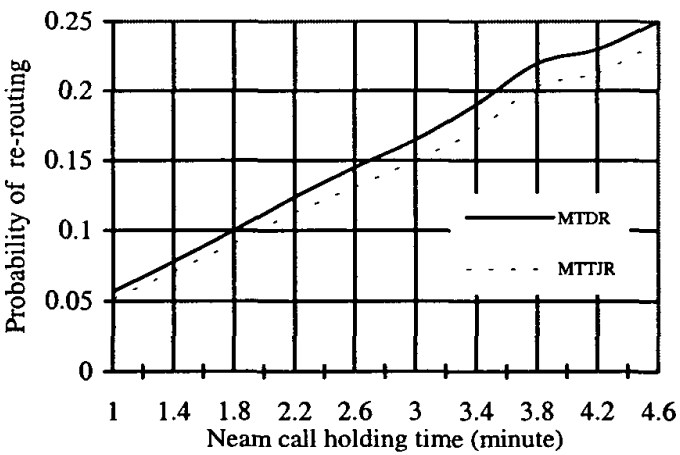

Fig. 6 The probability of call re-routing using MDTR and MTTJR

Communication Systems," IEEE Journal on Selected Areas in Communications, Vol. 13, No. 2, 458-464, 1995.

[9] Hu Jianhao, Wu Shiqi and Feng Gang, "Constellation Design for a LEO Mobile Satellite Communication System of China," ACTA ELECTRONICA SINICA, Vol.24, No.7, 16-21, 1996.

[10] Joaquin Restrepo and Gerard Maral, "Cellular Geometry for World Wide Coverage By non-GEO Satellite Suing 'Earth Fixed Cell'," Space Communication, No. 3, 1996.

[11] Wu Shi Qi, etal. "Technique and Economic Analysis for the Mobile Satellite Communication System," Technology and Development, No.5, 1995. 